\title{
A SIMPLE METHOD FOR DETERMINING ASYMPTOTIC FORMS OF NAVIER-STOKES SOLUTIONS FOR A CLASS OF LARGE REYNOLDS NUMBER FLOWS
}

\section{By J. D. MurRay}

1. Introduction. The problern of finding uniformly valid asymptotic solutions to the Navier-Stokes equaticn has been studied particularly intensively in the past ten years. Goldstein (1956), (1960) suggested a first consistent extension to the Blasius boundary layer solution for the incompressible flow past a semiinfinite flat plate. $\dagger$ Murr'y (1965a) established the validity of Goldstein's solution up to the term of $O\left(\xi^{-3}\right)$, where $\xi$ is the conventional parabolic coordinate stretched by $(U / \nu)^{\frac{1}{2}}$ where $U$ is the free stream velocity and $\nu$ is the kinematic viscosity, by showing that certain constraints were met. In the low Reynolds number range the method of matched expansions was formally developed and applied to a variety of problems. An account of this method with a selected bibliography has been given by Van Dyke (1964a). A series of papers by Van Dyke (1962a, 1962b, 1964b) has laid the foundation for a rigorous approach in the large Reynolds number range.

Although systematic methods are available theoretically for finding asymptotic solutions, it can still be very difficult in practice to do so. In the case of the semi-infinite flat plate solution very careful reasoning was involved before the final form for the stream function, $\psi$, was found. An account of this is given by Goldstein (1960). It is clear, in this example, that once the form of $\psi$ near the plate, or rather in the singular perturbation region, was found, the major difficulty of the problem was overcome. It is the purpose of this paper to suggest a method which will give reasonable and possible asymptotic forms for the solutions, in the first instance, to a class of problems consisting of flows past semiinfinite cylinders in a direction parallel to the generators. The solutions are for the region where the Reynolds number based on the radius or the equivalent length in non-circular cylinders is large compared with $z_{1} / a$ where $z_{1}$ is the axial coordinate measured from the leading edge and $a$ is the cylinder radius or equivalent length.

The method may be described as a modified 'modified Oseen' method. Basically the solution of the boundary layer Oseen equation is taken, or even an approximate form of it in some cases, and $U$, in this solution, is replaced by a function of $z_{1}, U$ and $\nu$ where this function is to be determined, ordinarily in asymptotic form. The function of $z_{1}, U$ and $\nu$ is determined by substituting the modified boundary layer Oseen solution into the full Navier-Stokes equation and integrating over an appropriate range of the variable other than $z_{1}$, weighting the integrand if necessary for convergence. An ordinary differential equation for the function to be determined is obtained and, in the examples considered, an asymptotic expansion of the function can be found fairly simply. With this

t See the footnotes below regarding equns. (23) and (46). 
asymptotic solution a form for the stream function can easily be deduced as illustrated in $\$ \$ 2,3$.

The same starting point is, of course, obtained from the equivalent Rayleigh solution for the impulsive motion problems. The time, $t$, is replaced not by $z_{1} / U$ as was done by Rayleigh (1911) (to approximate the solution for the flow past a semi-infinite flat plate) but by a function of $z_{1}, U$ and $\nu$. Thus, the method could also be described as a modified 'Rayleigh method'.

The motivation for the method is that the Oseen equation is a valid first approximation to the exact Navier-Stokes equation over part of the field of flow. It is reasonable, therefore, to try and use the solution of it as a type of generating function in conjunction with the Navier-Stokes equation. The type of integration of the Navier-Stokes equation used should produce the type of singularity in the axial coordinate that could be expected to appear in the exact solution. As shown below, it seems to do so.

The method is applied first to the flow past a semi-infinite flat plate and the first three asymptotic terms are given correctly as compared with the Goldstein (1960) form for the stream function. Higher order terms are not given exactly but their character is indicated. The problem of the possible origin displacement which is specifically suggested by this method does not affect the comparison, as is shown below by means of a simple coordinate transformation. As a biproduct of this method the first term in the local skin friction coefficient is given to within $4 \%$ of the Blasius solution with comparable accuracy in other quantities of interest. The connection with the modified Oseen method given initially by Lewis and Carrier (1949) is clear in that the first term is the same as if $z_{1} / U$ were replaced by $z_{1} / c U$ where $c$ is a constant; this, in fact, is what they did. Their constant is a possible first term in the asymptotic expansion of the function of $z_{1}, U$, and $\nu$ introduced in this method. By a possible solution is meant that it is the case where the origin displacement is taken to be zero. Lewis and Carrier (1949), to determine $c$, required their approximate solution to be such that the difference between the exact and approximate form of the momentum terms, when suitably integrated, should be zero. As seen below the same result is given by integrating the boundary layer equations with the approximate solution inserted. Recently Carrier (1965) used the Blasius form of boundary layer equations to determine $c$. This connection accounts for the term, modified 'modified Oseen' method, and provides further motivation for the method developed here.

The problem of axial flow past a semi-infinite circular cylinder is considered in $\$ \$ 3,4$. Any difficulties in extending the analysis to other cross sections will be manipulative; in principle, no difficulty arises. The final form suggested for the stream function is an asymptotic solution for the full Navier-Stokes equation and not simply for the boundary layer equations for which, as far as the author is aware, all previous studies of this problem have been made. The form found is for large Reynolds number, Re, based on the cylinder radius and for large $\operatorname{Re}\left(z_{1} / a\right)$. It differs fundamentally from those previously given for the boundary layer equations and suggests that the solution, which hitherto has been used for 
comparison of approximate methods, and referred to as the exact solution may not be so exact! The solution, as given in $\$ 3, \mathrm{Eq}$. (45), is a type of dual series involving both a Reynolds number expansion and a coordinate expansion.

Although the analysis of the axial flow past a semi-infinite cylinder is not the main purpose of this paper, a brief survey of some of the pertinent literature on this subject will be given since a new result is presented here. A more complete list is given by Rosenhead (1963).

Atkinson and Goldstein in 1933 (see Goldstein (1938)) considered entry flow into a circular pipe by introducing a series expansion in powers of $\left(z_{1} v / a^{2} U\right)^{\frac{1}{2}}$ times functions of the variable $\left[\left(a^{2}-r_{1}^{2}\right) / 4 a\right]\left(U / z_{1} \nu\right)^{\frac{1}{2}}$, where $r_{1}$ is the radial coordinate and $U, z_{1}, a, \nu$ are as above. They used the boundary layer equations.

The method of Atkinson and Goldstein with the change in sign in the second of the above variables was essentially that used by Seban and Bond (1951) to study the boundary layer on the outside of a cylinder in an axial flow. Kelly (1954) made some numerical corrections to Seban and Bond's work. The solution by Seban, Bond and Kelly has been used, and referred to, as the exact solution for comparison in connection with suggested approximate methods. The solution given in $\S \S 3,4$ below suggest that, beyond their second term, the Seban, Bond and Kelly description is probably not a correct solution of the Navier-Stokes equation.

Glauert and Lighthill (1955) considered the same problem and, for the solution near the leading edge, used a Polhausen technique which requires a very careful choice of the velocity profile used. It compares well with the solution of Seban, Bond and Kelly. The same remarks above on the latter solution also hold for that of Glauert and Lighthill. In the same paper Glauert and Lighthill also considered the solution of the boundary layer equations in the region where the boundary layer is large compared with the cylinder radius, by expanding the stream function in inverse powers of $\log \left(4 \nu z_{1} / U a^{2}\right)$. Stewartson (1955) independently considered this latter problem with the same results. The boundary layer solution they found is consistent in this region of large $z_{1} / a$ with the results given by Batchelor (1954) in his study of the flow resulting from the impulsive motion of the infinite cylinder parallel to its generators. He used Rayleigh's (1911) method to relate his work to the axial flow problem.

Seban and Bond's (1951) analysis and Glauert and Lighthill's (1955) technique were directly extended by Cooke (1957) and by Varley (1957) to cylinders of arbitrary cross-section. Their suggested form and magnitude of terms higher than the second, in the region of the flow field where the boundary layer is small compared with the radius, are also probably wrong. Unlike the solution suggested below, all of these solutions cannot give the correct first order correction to the Blasius solution for the flat plate as the limit as the cylinder radius tends to infinity under the usual limiting process.

The use of Rayleigh's (1911) method has been criticized because of its poor comparison with the Blasius solution in the semi-infinite flat plate problem. The forthcoming analysis or comparison with the modified Oseen method when $U$ is replaced by $c U$, with $c$ appropriately chosen, shows that Rayleigh's method 
can be modified simply, to give fairly good agreement with the Blasius solution. Sowerby and Cooke (1953), independently to Lewis and Carrier, introduced $c U$ for $U$ in applying the Rayleigh method to the axial flow past a circular cylinder, and chose a value for $c$ so that the first term in this solution gave exactly the same value for the local skin friction coefficient as the Blasius solution. They then used this value of $c$ in higher order terms from Rayleigh's method but poor comparison was found on comparison with Seban and Bond's (1951) higher order terms. This is to be expected when one considers the implication of $c$ in $c U$. For each position $z_{1}$ from the leading edge there is clearly an appropriate value for $c$. Near the leading edge where the boundary layer is thin a value for $c$ which results in the first term from Rayleigh's method giving the same skin friction as the Blasius solution is appropriate. Higher order terms allow for the solution to hold farther from the leading edge in which region the boundary layer is thicker. At a position in this region a higher value for $c$, than that used in the solution nearer the leading edge, would thus be appropriate. Eventually in the region far downstream from the leading edge where the boundary layer is very large (compared with the cylinder radius) a value for $c$ near unity would be appropriate. In this case the strict application of Rayleigh's method, that is $c=1$, should give a reasonably good approximate answer. The solution in this region given by Batchelor (1954) using this has in fact been verified by Glauert and Lighthill's (1955) solution. However, even if a systematic method for choosing a numerical value for $c$ to be set in the second term from the Rayleigh-type solution near the leading edge is used to improve the second term, the correct third and higher terms cannot be obtained correctly in form (or magnitude) by the use of specific values for $c$ (see $\$ \S 2,3$ which show in effect that the second order approximation to $c$ is not a constant).

It appears, therefore, that the use of this averaging of the full Navier-Stokes equation with a modified solution of the boundary layer Oseen equations produces the expected type of singularities and the form of some of the terms in the asymptotic solution to a class of large Reynolds number flows. No attempt is made here at any rigorous mathematical justification for the method. The justification for it, at this stage, is that consistent solutions are found when comparison is made with the flat plate solution of Goldstein (1960) and Murray (1965a). Further, in the axial flow problem, the suggested stream function when substituted in the full Navier-Stokes equation, shows that a self-similar solution is possible and that it reduces to the correct $f$ flat plate solution under the usual limit. Justification in particular for the modified Oseen method is given by the close numerical comparison that is found with its use in specific problems. Weinbaum (1964) used it to obtain good results for the natural convection in a horizontal circular cylinder. In this case the $c$ is replaced for convenience by $c / r$ where $r$ is the radial coordinate measured from the cylinder center. Some examples and developments of the modified Oseen technique (as well as other approximate methods) are given by Carrier (1965). The weighting method is also described. The objective there, however, is different from ours in that good

$\uparrow$ See the footnotes below af ter equns. (23) and (46) regarding this solution. 
numerical approximations to the first term only are sought: in this paper it is the asymptotic forms which are required.

The ordinary differential equations resulting from these solutions produce a general linear operator (see \$4) which has occurred in a large number of problems. This seems to suggest that it is a fundamental operator in a class of selfsimilar solutions to the Navier-Stokes equations.

A further result which appears explicitly in this work is that the class of problems similar to those discussed below can be indeterminate to the extent of a displacement of the origin of the coordinates parallel to the body generators. This displacement is a function of $\nu$. This indeterminacy affects all terms in the solution and is a result of not knowing the solution near the origin (or leading edge). This indeterminacy is different from that discussed by Goldstein (1960) and Murray (1965a). A similar origin displacement is, of course, possible in this latter solution since the leading edge is not covered by the solution.

The application of the idea given here may not necessarily be restricted to the situations described below, nor to this class of flows, since the main point is that the use of integrals of the Navier-Stokes operator on a function related to the problem in question seems to produce at least some of the singularities that may be expected in the exact solution. The author intends to study this further. The method may also be relevant to singular partial differential equations in general.

2. Illustration of the method: Flow past a semi-infinite flat plate. The problem considered is the flow of an incompressible viscous fluid past a two-dimensional semi-infinite flat plate. The free stream velocity in the $x_{1}$-direction is $U$, the plate is $y_{1}=0, x \geqq 0$, where $x_{1}, y_{1}$ are Cartesian coordinates, $u_{1}, v_{1}$ are the velocity components, $p_{1}$ is the pressure, $\rho$ is the density, $\psi_{1}$ is the stream function, and $\nu$ is the kinematic viscosity.

The solution to the boundary layer Oseen equation

$$
U u_{1 x_{1}}=\nu u_{1_{y_{1} y_{1}}},
$$

subject to $u_{1}=0$ on $y_{1}=0, x_{1}>0$ and $u_{1} \rightarrow U$ as $y_{1} \rightarrow \infty, x_{1}>0$ is

$$
u_{1}=U \operatorname{erf} \frac{y_{1}}{2}\left(\frac{U}{\nu x_{1}}\right)^{\frac{1}{2}} .
$$

The velocity component $v_{1}$ is obtained from the continuity equation.

Lewis and Carrier (1949) considered the flow past a flat plate using the Oseen equations but they introduced a constant $c$ by writing $c U$ for $U$ in the equations. The constant $c$, which is expected to be less than unity, was determined by requiring the solution for $u_{1}$, as a function of $c$, to satisfy some integral of the difference between the exact and approximate form of the $u_{1}$-convective terms. That is, they required their $u_{1}$ to satisfy

$$
\int_{0}^{\infty} u_{x_{1}}\left(u_{1}-c U\right) d y_{1}=0
$$

or some weighted form of this. Effectively (as seen below) this requirement is 
the same as requiring (2), and the resulting $v_{1}$, with $c U$ for $U$, to satisfy

$$
\int_{0}^{\infty}\left(u_{1} u_{1 x_{1}}+v_{1} u_{1 y_{1}}-\nu u_{1 y_{1} y_{1}}\right) d y_{1}=0 \text {. }
$$

In this particular case $c=\sqrt{2}-1$. As mentioned in $\$ 1$ Carrier (1965) uses the Blasius equation to find this value of $c$. His starting function (and method) is slightly different.

If the Blasius solution is used for comparison, the local skin friction coefficient given by (2) is approximately $70 \%$ too high, while that with $(\sqrt{2}-1) U$ replacing $U$ is only $9 \%$ too high. If the integrand in (3) is weighted with $u_{y_{1}}$ then $c=\sqrt{3}-\sqrt{2}$ in which case the local skin friction coefficient is within $4 \%$ (lower) of the Blasius value. By allowing $c$ to be a special function of $x$, involving two undetermined constants, Carrier can in fact very closely reproduce the Blasius function; but not higher order terms in the solution.

The basic simple idea behind the method suggested in this paper is to replace $U$, or rather $\left(U / \nu x_{1}\right)$ in $(2)$ by $\gamma_{1}\left(x_{1}, \nu\right)^{*}$ and to find a form for $\gamma_{1}$ by integrating the full Navier-Stokes equation, weighted if necessary to insure convergence, in a manner similar to (3). An ordinary differential equation for $\gamma_{1}$ results, from which an asymptic solution can be found for small $\nu$. From this an asymptotic form for the stream function can be found easily. Simply, the motivation is that $U u_{1 x_{1}}$ is not a sufficiently accurate approximation to $u_{1} u_{1 x_{1}}+v_{1} u_{1 y_{1}}$ and so we replace $U$ by a function of $x_{1}, \nu$, and $U$ in an attempt to approximate the exact asymptotic form solution by taking into account the effect of the correct momentum terms in an integral form.

It is convenient to non-dimensionalize the equations of motion by writing

$$
\begin{array}{lll}
x=x_{1} U / \nu, & y=y_{1} U / \nu, & u=u_{1} / U \\
v=v_{1} / U, & p=p_{1} / \rho U^{2}, & \psi=\psi_{1} / \nu, \quad \gamma=\gamma_{1} \nu / U,
\end{array}
$$

in which case the equations become

$$
\begin{aligned}
u u_{x}+v u_{y} & =-p_{x}+\left(u_{x x}+u_{y y}\right), \\
u u_{x}+v v_{y} & =-p_{y}+\left(v_{x x}+v_{y y}\right), \\
u_{x}+v_{y} & =0 .
\end{aligned}
$$

Elimination of $p$ from (5) and (6) gives the Navier-Stokes equation as

$$
\begin{aligned}
L(u, v)=u u_{x}+v u_{y} & -v v_{x}-\left(u_{y y}+2 u_{x x}\right) \\
& -\frac{\partial}{\partial x}\left[\int_{0}^{y}\left(u v_{x}-v_{x x}\right) d y+\left\{\left(v_{y}\right)_{y=0}-(p)_{y=0}\right\}\right]=0,
\end{aligned}
$$

where $L(u, v)$ is defined by $(8)$.

* This is equivalent to writing a general function of $x_{1}, U$ and $\nu$ in place of the constant $c$ in the modified Oseen method. 
It is suggested that

$$
u=\operatorname{erf} \gamma(x) y, \quad \psi=y \operatorname{erf} \gamma y-\frac{1}{\gamma \sqrt{ } \pi}\left[1-\exp \left(-\gamma^{2} y^{2}\right)\right]
$$

(the latter equation being obtained using $(7)$ ) will result in an approximate asymptotic form for large $x$ for the solution to the full Navier-Stokes equation (8) when (9) is required to satisfy

$$
\int_{0}^{\infty} u_{y} L(u, v) d y=0 .
$$

The weighting function $u_{y}$ in (10) is used so that every term in the integral (10) converges at the upper limit; its inclusion in (3) was not necessary but as shown above did not, as was to be expected, change the answer qualitatively. Throughout we consider $\gamma(x) \geqq 0, y \geqq 0$.

Substitution of (9) in (10) gives, after some simple but slightly tedious algebra, the following ordinary differential equation for $\gamma(x)$ :

$$
c \gamma^{\prime} / \gamma^{2}+2 \gamma=F_{1}(\gamma)-F_{2}(\gamma), \dagger
$$

where

$$
\begin{aligned}
& F_{1}(\gamma)=2\left(\gamma^{\prime \prime} / \gamma^{2}-\gamma^{\prime 2} / \gamma^{3}\right)+a_{1} \gamma^{\prime \prime \prime} / \gamma^{4}+a_{2} \gamma^{\prime} \gamma^{\prime \prime} / \gamma^{5}+a_{3} \gamma^{33} / \gamma^{6} \\
& F_{2}(\gamma)=b_{1} \gamma^{\mathrm{iv}} / \gamma^{4}+b_{2} \gamma^{\prime} \gamma^{\prime \prime \prime} / \gamma^{5}+b_{3} \gamma^{\prime \prime 2} / \gamma^{5}+b_{4} \gamma^{\prime 2} \gamma^{\prime \prime} / \gamma^{6}+b_{5} \gamma^{4} / \gamma^{7}
\end{aligned}
$$

and

$$
\begin{aligned}
& c=\sqrt{3}-\sqrt{2}, \quad a_{1}=1+\pi / 12-\sqrt{2}, \\
& a_{2}=-7-3 \pi / 4+11 / \sqrt{2}+7 / 2 \sqrt{3}, \\
& a_{3}=8+\pi-19 / 2 \sqrt{2}-8 / \sqrt{3}, \\
& b_{1}=-1+\pi / 4, \quad b_{2}=10-3 \pi, \quad b_{3}=15 / 2-9 \pi / 4, \\
& b_{4}=-33+9 \pi, \quad b_{5}=35-21 \pi / 2 .
\end{aligned}
$$

The primes denote differentiation with respect to $x$. If the dimensional form of the equations had been used, (11) would be for $\gamma_{1}\left(x_{1}, \nu\right)$ and $\nu^{2}$ would multiply $F_{1}\left(\gamma_{1}\right)$ and $\nu^{4}$ would multiply $F_{2}\left(\gamma_{1}\right)$.

Since we are interested in asymptotic forms for large $x$, that is a coordinatetype expansion, we write $\gamma$ in the form of an asymptotic series

$$
\gamma=\gamma_{0}+\gamma_{1}+\gamma_{2}+\cdots,
$$

which on substitution in (11) gives for $\gamma_{0}$,

$$
c \gamma_{0}^{\prime} / \gamma_{0}^{2}+2 \gamma_{0}=0 \text {. }
$$

The solution of (13) is

$$
\gamma_{0}=\frac{1}{2}[A+x / c]^{-\frac{1}{2}},
$$

$\dagger$ The $F_{1}, F_{2}$ are functions of $\gamma$ and its derivatives. 
where $A$ is a constant to be determined from the leading edge solution (or boundary condition) which is at present unknown.

An indication of the nature of $A$ in dimensional form as $A_{1}$ can perhaps be more clearly seen if we put $\gamma_{0}$ for $\gamma$ in (9), which in dimensional form gives

$$
u_{1}=U \operatorname{erf}\left\{\frac{1}{2} y_{1}\left[\nu x_{1} / c U+\nu A_{1}(\nu)\right]^{-\frac{1}{2}}\right\} .
$$

Although the expansion (12) is valid only for large $U x_{1} / \nu$, if we consider the last equation to hold up to $x_{1}=0$, then with $y_{1} \neq 0, x_{1}=0$,

$$
u_{1}=U \operatorname{erf} y_{1} / 2 \sqrt{A_{1}(\nu) \nu} \text {. }
$$

If this holds, $A_{1}(\nu)$ must be such that $\lim A_{1}(\nu)=0$ as $\nu \rightarrow 0$, otherwise the first order solution for $u_{1}$ above for $\nu \rightarrow 0$ would be independent of $x_{1}$. Thus a first order solution has $A \equiv 0$ and a modified form of (2) is obtained with $(\sqrt{3}-\sqrt{2}) U$ for $U$. Of course the solution is not expected to hold at $x=0$, just as the Blasius solution is not expected to do so. At this point $v$, from (9), is infinite. As seen below $\gamma_{1}, \gamma_{2}, \cdots$ are functions of $\gamma_{0}$ so the influence of $A$ (or the leading edge) is felt in each term of the expansion. It represents, in a sense, an unknown (at this stage) displacement of the origin of the coordinate system parallel to the $x_{1}$-axis. Such a displacement is also possible in the Goldstein solution. If we introduce

$$
X_{1}=x_{1}+c U A_{1}(\nu),
$$

(5)-(8) have $X$ for $x$ and the resulting solution for $u_{1}$ is

$$
u_{1}=U \operatorname{erf} \frac{y_{1}}{2}\left(\frac{c U}{\nu X_{1}}\right)^{\frac{1}{2}} \text {. }
$$

This is valid only for large $U X_{1} / \nu$. If it were valid at $X_{1}=0$ it would give $u_{1}=U$ at $X_{1}=0, y_{1} \neq 0$ but it would also give $v$ infinite. $A$ is, of course, positive and is an estimate of the distance of the leading edge influence in the flow upstream of $x_{1}=0$ for the leading term.

To proceed to higher order terms in $\gamma$ it is easy to see that $\gamma_{0}$ from (14) is small when $x$ is large and the right side of (11) is of higher order than the left and $F_{2}$ is of higher order than $F_{1}$. In fact, from (14),

$$
\gamma_{0}^{\prime}=\frac{-2}{c} \gamma_{0}^{3}, \quad \gamma_{0}^{\prime \prime}=\frac{12}{c^{2}} \gamma_{0}^{5}, \quad \text { etc. }
$$

and so

$$
\begin{aligned}
F_{1}\left(\gamma_{0}\right) & =a_{4} \gamma_{0}^{3}, \quad F_{2}\left(\gamma_{0}\right)=b_{5} \gamma_{0}^{5}, \\
a_{4} & =\frac{1}{c^{2}}\left[16-\frac{8}{c}\left(15 a_{1}+3 a_{2}+a_{3}\right)\right], \quad b_{5}=\frac{8}{c^{4}}(97-45 \pi) .
\end{aligned}
$$

Neither of $a_{4}, b_{5}$ is zero.

The equation for $\gamma_{1}$ from (11) and (12) is thus

$$
c \gamma_{1}^{\prime} / \gamma_{0}^{2}+6 \gamma_{1}=a_{4} \gamma_{0}^{3}
$$


the solution of which using (13) is

$$
\gamma_{1}=B \gamma_{0}^{3}-\frac{1}{2} a_{1} \gamma_{0}^{3} \log \gamma_{0},
$$

where $B$ is a further undetermined constant.

Similarly, from (11), (12), (13), (16) and (17)

$$
\gamma_{2}=\gamma_{0}^{5}\left[H_{1}(B)+H_{2}(B) \log \gamma_{0}+\frac{3}{20} a_{4}^{2}\left(\log \gamma_{0}\right)^{2}\right],
$$

where $H_{1}, H_{2}$ are known functions of $B$. A complementary function $\gamma_{0}^{3}$ is also given by the equation for $\gamma_{2}$, and all higher terms, but it is incorporated in the undetermined constant $B$ in $\gamma_{1}$.

Although a form for the stream function $\psi$ is suggested by $u_{y}=0$ at $y=0$ it is better to consider $\psi$ from (9), which may be written as

$$
\psi=\frac{1}{\gamma} f(\gamma y)
$$

where $f(\gamma y)$ is a function of $\gamma y$ alone. With $\gamma$ expanded as in (12), (19) may be expanded to give

$$
\begin{aligned}
\psi=\frac{1}{\gamma_{0}}\left[f\left(\gamma_{0} y\right)+\right. & \frac{\gamma_{1}}{\gamma_{0}}\left\{\gamma_{0} y f^{\prime}\left(\gamma_{0} y\right)-f\left(\gamma_{0} y\right)\right\} \\
+\frac{\gamma_{1}^{2}}{\gamma_{0}^{2}} & \left\{\left[\left(\gamma_{0} y\right)^{2} / 2+\frac{\gamma_{2} \gamma_{0}}{\gamma_{1}^{2}}\left(\gamma_{0} y\right)\right] f^{\prime \prime}\left(\gamma_{0} y\right)\right. \\
& \left.\left.\quad-\gamma_{0} y f^{\prime}\left(\gamma_{0} y\right)+\left(1-\frac{\gamma_{2} \gamma_{0}}{\gamma_{0}^{2}}\right) f\left(\gamma_{0} y\right)\right\}+\cdots\right] .
\end{aligned}
$$

Since $\gamma_{1}, \gamma_{2}$, etc. from (17) and (18) are functions of $\gamma_{0}$ only, (20) is an equation with variables $\gamma_{0}$ and $\gamma_{0} y$ only. If we set $A=0$ in (14) then $\gamma_{0}^{-1}$ is proportional to $R_{x_{1}}$, the Reynolds number based on $x_{1}$. We are thus led to introduce the natural variables $\xi, \eta$, in the case when $A=0$, by

$$
\xi=\left(U x_{1} / \nu\right)^{\frac{1}{2}}, \quad \eta=\frac{1}{2} y_{1}\left(\frac{U}{\nu x_{1}}\right)^{\frac{s}{3}}
$$

which are proportional to $\gamma_{0}^{-1}$ and $\gamma_{0} y$ respectively. However, it is not necessary to set $A=0$ as shown above with the transformation (15) redefining the $x_{1}$ coordinate. The appropriate $\xi, \eta$ are then

$$
\xi=\left(\frac{U X_{1}}{\nu}\right)^{\prime}, \quad \eta=\frac{1}{2} y_{1}\left(\frac{U}{\nu X_{1}}\right)^{\frac{1}{2}} .
$$

A suggested form for the stream function is thus, from (17), (18), (20) and (21) or (22),

$$
\begin{aligned}
\psi=\xi\left[f_{0}(\eta)+\frac{1}{\xi^{2}}\right. & \left\{f_{2}(\eta)+g_{2}(\eta) \log \xi\right\} \\
& \left.+\frac{1}{\xi^{4}}\left\{f_{3}(\eta)+g_{3}(\eta) \log \xi+h_{3}(\eta)(\log \xi)^{2}\right\}+\cdots\right] .
\end{aligned}
$$


The first three terms in (23) are those given by Goldstein (1960) $\dagger$ and computed by Murray (1965a) $\dagger$ : parabolic coordinates are used there in place of the $\xi, \eta$ above. Parabolic coordinates are clearly suggested by (21) and (22). The fourth and higher terms in (23) are not correct in form compared with Goldstein's (1960) solution in that the power of $\xi$ in the $f_{3}, g_{3}, h_{3}$ bracket should be $\xi^{-2}$ and not $\xi^{-3}$ as in (23). The form and powers of the logarithms are the same, however.

In (20), the $\gamma_{1}$ (from (17)) contains an undetermined constant $B$ in the nonlogarithm term, which suggests that such an indeterminacy might appear in finding $f_{2}(\eta)$ in (23). As shown by Goldstein (1960) and Murray (1965a) this is in fact the case. Further, a form which could be suggested from $(20)$ is $g_{2}(\eta)=$ $a\left(\eta f_{0}^{\prime}-f_{0}\right)$ where $a$ is some constant which can be determined. Goldstein (1960) shows that $g_{2}$ is in fact proportional to $\left(\eta f_{0}^{\prime}-f_{0}\right)$ and Murray (1965a) shows that $a=1.569$.

A form for $\psi$ for large $\xi$, such as (23), is not sufficient to find the complete solution. A potential solution must be found into which $\psi$ merges for large $\eta$. This potential solution gives the boundary conditions on the functions of $\eta$ for large $\eta$. These functions of $\eta$ all have double zeros at the origin. Goldstein (1960) deduced the form of $\psi$ for higher order terms than the first, from a knowledge of the exterior potential flow. When the foregoing method is used, the potential flow would be suggested by the foregoing result. What is equally important, of course, is that it must be verified that the suggested $\psi$ is consistent with a possible potential flow.

This example illustrates the method and its comparative success in predicting the correct form of the stream function for large $x$. It also gives a good quantitative value for the first term in such quantities as the local skin frinction coefficient and the displacement thickness. It does not give the correct fourth and higher terms as to the power of $\xi$. The second and third terms are invariably the most difficult to obtain and if they can be determined higher order terms in many cases may be inferred from them, particularly with the experience gained from a knowledge of the semi-infinite flat plate solution of Goldstein (1960) $\dagger$ and Murray $(1965 a) \dagger$. In this latter solution the origin displacement found above is suggested by Goldstein's analysis since it does not cover the region near the leading edge.

3. Incompressible axial flow past a semi-infinite circular cylinder. As was discussed in the introduction, the problem of this section has been studied fairly extensively before but such studies have been restricted to similarity solutions of the boundary-layer equations of motion. We consider here the complete Navier-Stokes equations and we look for an asymptotic description of the flow

$\dagger$ It is still possible that this solution is not sufficiently general. The various functions of $\eta$ in (23) all satisfy ordinary differential equations with the operator $L_{n}$ (see (50) below). There is still the possibility of eigensolutions for non-integral $n=\lambda$, say, being included in the form $\Sigma_{\lambda} f_{\lambda} / \xi^{\lambda}$ where the $f(\eta)$ are exponentially small for $\eta$ large. These are essentially perturbations about the Blasius solution, some of which have been computed by Libby and Fox (1963). 
which is valid outside the cylinder: we hope to be guided by that found in $\$ 2$ for the flow past a semi-infinite flat plate.

We consider here the viscous axially symmetric flow past a semi-infinite circular cylinder of radius $a$. The velocity components in the axial, $z_{1}$, and radial, $r_{1}$, directions respectively are $w_{1}, u_{1}$ : the free stream velocity is $U$ in the axial direction. The origin of the coordinate system is on the axis at the leading edge of the cylinder. We seek an asymptotic solution to the axially symmetric NavierStokes equations of motion, namely (25)-(27) below, valid in $r_{1} \geqq a, z_{1}>0$, such that $w_{1}, u_{1}$ are zero on $r_{1}=a, z_{1} \geqq 0$ and $w_{1} \rightarrow U, u_{1} \rightarrow 0$ as $r_{1} \rightarrow \infty$.

There are now two lengths in the problem, the cylinder radius, $a$, and the viscous length $\nu / U$. Introduce the non-dimensional quantities

$$
\begin{aligned}
& r=r_{1} / a, \quad z=z_{1} / a, \quad R=r-1, \quad p=p_{1} / \rho U^{2}, \\
& w=u_{1} / U, \quad u=u_{1} / U, \quad \operatorname{Re}=U a / \nu, \quad \psi=\downarrow_{1} / U a^{2} .
\end{aligned}
$$

The surface of the cylinder lies in $R=0, z \geqq 0$. The non-dimensional equations of motion are given by

$$
\begin{aligned}
w w_{z}+u w_{R} & =-p_{z}+\frac{1}{\operatorname{Re}}\left[w_{R R}+\frac{1}{r} w_{R}+w_{z z}\right], \\
w u_{z}+u u_{R} & =-p_{R}+\frac{1}{\operatorname{Re}}\left[u_{R R}+\frac{1}{r} u_{R}-\frac{1}{r^{2}} u+u_{z z}\right], \\
(r u)_{R}+(r w)_{z} & =0,
\end{aligned}
$$

where $w=0=u$ on $R=0, z \geqq 0$ and $w \rightarrow 1, u \rightarrow 0$ as $R \rightarrow \infty$.

Eliminating the pressure from (25), (26) gives

$$
\begin{aligned}
M(w, u)=w w_{z}+ & u w_{R}-u u_{z} \\
-\frac{1}{\operatorname{Re}} & {\left[w_{R R}+\frac{1}{r} w_{R}+2 w_{z z}-\left(w_{R R}+\frac{1}{r} w_{R}\right)_{R=0}\right] } \\
& -\frac{\partial}{\partial z}\left[\int_{0}^{R} w u_{z} d R-\frac{1}{\operatorname{Re}} \int_{0}^{R} u_{z z} d R+\left(u_{R}\right)_{R=0}\right]=0,
\end{aligned}
$$

where $M(w, u)$ is defined by $(28)$.

A direct comparison with $\$ 2$ suggests that a suitably modified boundary layer Oseen solution should be used for $w$ just as (9) was used in the previous section. Thus, we choose [see Carslaw and Jaeger (1947)]

$$
w=-\frac{2}{\pi} \int_{0}^{\infty}\left\{\frac{J_{0}(k r) Y_{0}(k)-J_{0}(k) Y_{0}(k r)}{J_{0}^{2}(k)+Y_{0}^{2}(k)}\right\} \exp \left(-\frac{z k^{2}}{\mathrm{Re}}\right) \frac{d k}{k},
$$

which for small $z /$ Re may be written as

$$
w=1-r^{-\frac{1}{2}} \operatorname{erfc} \frac{R}{2}\left(\frac{\operatorname{Re}}{z}\right)^{\frac{1}{2}}+\frac{R-1}{4 r^{3}}\left(\frac{z}{\operatorname{Re}}\right)^{\frac{1}{2}} i \operatorname{erfc} \frac{R}{2}\left(\frac{\operatorname{Re}}{z}\right)^{\frac{1}{2}}-\cdots
$$

$\dagger$ The $J_{0}$ and $Y_{0}$ are the usual Bessel functions. 
The method in this paper suggests that it is not necessary to use (29) with $\frac{1}{2}(\mathrm{Re} / z)^{\frac{3}{2}}$ replaced by $\gamma(z, \mathrm{Re})$ in the equivalent integral to (10) (equation (33) below) but simply

$$
w=\operatorname{erf} \gamma(z, \mathrm{Re}) R .
$$

This is an approximation to the first two terms of $(30)$ for the region close to the cylinder. More importantly, however, $(31)$ is also a modified (with $\gamma(z, R e)$ ) solution of $w_{z}=(\mathrm{Re})^{-1} w_{R R}$, while (29) is the solution of the Oseen-linearized boundary layer equation (there is an extra term $\operatorname{Re} r^{-1} w_{R}$ on the right of the last equation).

The velocity components $w, u$ are given by (27) as

$$
w=\frac{1}{r} \psi_{r}, \quad u=-\frac{1}{r} \psi_{2},
$$

and so from (31) the stream function, $\psi$, is

$$
\begin{aligned}
\psi=[R \operatorname{erf} \gamma R+ & \left.\frac{1}{\gamma \sqrt{\pi}}\left\{\exp \left(-\gamma^{2} R^{2}\right)-1\right\}\right] \\
& +\left[\frac{1}{4 \gamma^{2}}\left(2 \gamma^{2} R^{2}-1\right) \operatorname{erf} \gamma R+\frac{R}{2 \gamma \sqrt{\pi}} \exp \left(-\gamma^{2} R^{2}\right)\right] .
\end{aligned}
$$

Again $\gamma \geqq 0, R \geqq 0$. The first square bracket is similar in form to (9). We now require $w, u$ to satisfy

$$
\int_{0}^{\infty} w_{R} M(w, u) d R=0,
$$

the equivalent of $(10)$ in $\$ 2$, where here $M(w, u)$ is given by (28). Not all of the integrals in (33) can be obtained easily in closed form. However, since we are interested, at this stage, in the range $\operatorname{Re} \gg z$, the function, $\gamma(z, \operatorname{Re})$, in (31) and (32) is large and asymptotic forms for the integrals can be found. Note that with $\operatorname{Re} \gg z$ the solution we shall obtain is that in which the boundary layer thickness is small compared with the cylinder radius.

Substitution of (32) into (33) gives, after even more tedious algebra, as the ordinary difterential equation for $\gamma(z, \mathrm{Re})$,

$$
c \gamma^{\prime} / \gamma^{2}+\frac{2}{\operatorname{Re}} \gamma=f_{1}(\gamma, \mathrm{Re})+f_{2}(\gamma, \mathrm{Re})+f_{3}(\gamma, \mathrm{Re})+\cdots,
$$

where $c=\sqrt{3}-\sqrt{2}$ as in $\S 2$, the prime denotes differentiation with respect to $z$, the higher $f$-terms are of small order in $\gamma$ and Re and

$f_{1}(\gamma, \operatorname{Re})=c_{1} \gamma^{\prime} / \gamma^{3}+c_{2} / \operatorname{Re}$,

$f_{2}(\gamma, \operatorname{Re})=d_{1} \gamma^{\prime \prime \prime} / \gamma^{4}+d_{2} \gamma^{\prime} \gamma^{\prime \prime} / \gamma^{5}+d_{3} \gamma^{\prime 2} / \gamma^{6}+\frac{1}{\operatorname{Re}}\left(d_{4} \gamma^{\prime 2} / \gamma^{3}+d_{5} / \gamma\right)$,

$f_{3}(\gamma, \operatorname{Re})=e_{1} \gamma^{\prime \prime \prime} / \gamma^{5}+e_{2} \gamma^{\prime} \gamma^{\prime \prime} / \gamma^{6}+e_{3} \gamma^{\prime 3} / \gamma^{7}+e_{4} \gamma^{\prime} / \gamma^{5}+\frac{1}{\operatorname{Re}}\left(e_{6} / \gamma^{2}\right)$, 
where $c_{\imath}, d_{\imath}, e_{i}, i \geqq 1$, none of which is zero, are given by

$$
\begin{aligned}
& c_{1}=\left(\sqrt{2} \tan ^{-1} \sqrt{2}-1\right) / \sqrt{\pi}, \\
& c_{2}=\sqrt{2 \pi}-2 \sqrt{\pi}, \quad c_{3}=c_{2}-\frac{2}{c} c_{1}, \\
& d_{1}=1+\pi / 12-\sqrt{2}, \quad d_{2}=-7-\frac{3}{4} \pi+6 \sqrt{2}+7 / 2 \sqrt{3}, \\
& d_{3}=8+\pi-19 / 2 \sqrt{2}-8 / \sqrt{3}, \quad d_{4}=3, \quad d_{5}=-1, \\
& d_{6}=-\frac{4}{c^{3}}\left(30 d_{1}+6 d_{2}+2 d_{3}-c d_{4}\right), \quad d_{7}=\frac{1}{3} c_{3}+\frac{2}{c} c_{1} c_{3}+d_{5}, \\
& e_{1}=\frac{1}{\sqrt{\pi}}\left(\frac{1}{2}-2 \sqrt{2} \tan ^{-1} \sqrt{2}-\sqrt{2} \tan ^{-1} 1 / \sqrt{2}\right), \\
& e_{2}=\frac{1}{\sqrt{\pi}}\left(-\frac{9}{2}+\frac{3}{4} \pi+19 \sqrt{2} \tan ^{-1} \sqrt{2}-9 \sqrt{2} \tan ^{-1} 1 / \sqrt{2}\right), \\
& e_{3}=\frac{1}{\sqrt{\pi}}\left(\frac{35}{12}-\frac{7}{4} \pi-27 \sqrt{2} \tan ^{-1} \sqrt{2}+\frac{23}{\sqrt{2}} \tan ^{-1} 1 / \sqrt{2}\right), \\
& e_{4}=\frac{1}{\sqrt{\pi}}\left(-\frac{1}{3}+\frac{1}{2 \sqrt{2}} \tan ^{-1} \sqrt{2}\right), \quad e_{5}=\frac{\sqrt{\pi}}{2 \sqrt{2}} \cdot
\end{aligned}
$$

It is convenient to write

$$
Z=\operatorname{Re} z, \quad \gamma(z, \operatorname{Re})=\operatorname{Re} \Gamma(Z, \operatorname{Re}),
$$

where, with Re large and $Z$ large, $\Gamma$ is small. Equation (34) now becomes $c \Gamma^{\prime} / \Gamma^{2}+2 \Gamma=\left[d_{1} \Gamma^{\prime \prime \prime} / \Gamma^{4}+d_{2} \Gamma^{\prime} \Gamma^{\prime \prime} / \Gamma^{5}+d_{3} \Gamma^{\prime 3} / \Gamma^{6}+d_{4} \Gamma^{\prime 2} / \Gamma^{7}+\cdots\right]$

$$
\begin{gathered}
+\frac{1}{\operatorname{Re}}\left[\left\{c_{1} \Gamma^{\prime} / \Gamma^{3}+c_{2}\right\}+\left\{e_{1} \Gamma^{\prime \prime \prime} / \Gamma^{5}+e_{2} \Gamma^{\prime} \Gamma^{\prime \prime} / \Gamma^{6}+e_{3} \Gamma^{3} / \Gamma^{7}\right\}+\cdots\right] \\
+\frac{1}{(\operatorname{Re})^{2}}\left[d_{5} / \Gamma+\cdots\right]+\frac{1}{(\operatorname{Re})^{3}}\left[e_{4} \Gamma^{\prime} / \Gamma^{5}+e_{5} / \Gamma^{2}+\cdots\right]+\cdots,
\end{gathered}
$$

where the prime denotes differentiation with respect to $Z$. With Re large, (38) suggests expanding $\Gamma$ in a simple series

$$
\Gamma=\Gamma_{0}(Z)+\frac{1}{\operatorname{Re}} \Gamma_{1}(Z)+\frac{1}{(\operatorname{Re})^{2}} \Gamma_{2}(Z)+\cdots
$$

In the dimensional form of $(11)$ in $\$ 2$ an expansion would have been in powers of $\nu^{2}$. Substitution of (39) into (38) gives equations for $\Gamma_{0}, \Gamma_{1}, \Gamma_{2}, \cdots$, where, for example,

$$
c \Gamma_{0}^{\prime} / \Gamma_{0}^{2}+2 \Gamma_{0}=d_{1} \Gamma_{0}^{\prime \prime \prime} / \Gamma_{0}^{4}+d_{2} \Gamma_{0}^{\prime} \Gamma_{0}^{\prime \prime} / \Gamma_{0}^{5}+d_{3} \Gamma_{0}^{\prime 3} / \Gamma_{0}^{6}+d_{4} \Gamma_{0}^{\prime 2} / \Gamma_{0}^{3}+\cdots .
$$

Equation (40) now suggests that $\Gamma_{0}(Z)$ should be expressed as an asymptotic 
coordinate expansion for large $Z$ in analogy to the expansion for $\gamma$ in $\$ 2$. We write

$$
\Gamma_{0}={ }_{0} \Gamma_{0}+{ }_{1} \Gamma_{0}+{ }_{2} \Gamma_{0}+\cdots,
$$

where from (40) we find that

$$
\begin{aligned}
& { }_{0} \Gamma_{0}=\frac{1}{2}[C+Z / c]^{-\frac{1}{2}}, \quad{ }_{1} \Gamma_{0}=D_{0} \Gamma_{0}^{3}-d_{60} \Gamma_{0}^{3} \log { }_{0} \Gamma_{0}, \\
& { }_{2} \Gamma_{0}={ }_{0} \Gamma_{0}^{5}\left[H_{3}(D)+H_{4}(D) \log { }_{0} \Gamma_{0}+\frac{3}{2} d_{6}^{2}\left(\log _{0} \Gamma_{0}\right)^{2}\right], \text { etc., }
\end{aligned}
$$

where $C$ (corresponding to $A$ in (14)) is an undetermined constant and represents a displacement of the origin of $z_{1}, D$ is another undetermined constant comparable to $B$ in (17), $H_{3}, H_{4}$ are known functions of $D$, and $d_{6}$ is given by (36). The series for $\Gamma_{0}$ is similar to that for $\gamma$ in \$2. This is expected, since for large $a$ (or small $z_{1}$ ) the cylinder approximates to a semi-infinite flat plate.

The equation for $\Gamma_{1}$ is obtained from (38) by equating the coefficients of the $1 /$ Re terms in (38). Again, we expand $\Gamma_{1}$ as a coordinate expansion

$$
\Gamma_{1}={ }_{0} \Gamma_{1}+{ }_{1} \Gamma_{1}+{ }_{2} \Gamma_{1}+\cdots
$$

and find that

$$
\begin{aligned}
& { }_{0} \Gamma_{1}=\frac{1}{3} c_{3}+{ }_{0} \Gamma_{0}^{2}\left[h_{1}+h_{2} \log { }_{0} \Gamma_{0}\right], \quad{ }_{1} \Gamma_{1}=E_{0} \Gamma_{0}^{3} \\
& { }_{2} \Gamma_{1}={ }_{0} \Gamma_{0}^{4}\left[H_{5}(D)+H_{6}(D) \log { }_{0} \Gamma_{0}+h_{3}\left(\log { }_{0} \Gamma_{0}\right)^{2}\right] \\
& { }_{3} \Gamma_{1}=E_{0} \Gamma_{0}^{5}\left[H_{7}(D)+h_{4} \log { }_{0} \Gamma_{0}\right], \text { etc. }
\end{aligned}
$$

where $h_{\imath}, i \geqq 1$ are known non-zero constants, $H_{5}, H_{6}, H_{7}$ are known functions of $D$ (equation (41)) and $E$ is another undetermined constant.

With an expansion for $\Gamma_{2}$ analogous to those for $\Gamma_{1}$ and $\Gamma_{0}$, we find that

$$
\begin{aligned}
& { }_{0} \Gamma_{2}=d_{7} / 4_{0} \Gamma_{0}, \\
& { }_{1} \Gamma_{2}={ }_{0} \Gamma_{0}\left[H_{8}(D)+h_{5} \log { }_{0} \Gamma_{0}\right], \quad{ }_{2} \Gamma_{2}=E h_{6} \Gamma_{0}^{2}, \\
& { }_{3} \Gamma_{2}={ }_{0} \Gamma_{0}^{3}\left[F+H_{9}(D) \log { }_{0} \Gamma_{0}+h_{7}\left(\log { }_{0} \Gamma_{0}\right)^{2}\right], \\
& { }_{4} \Gamma_{2}=E_{0} \Gamma_{0}^{4}\left[H_{10}(D)+h_{8} \log { }_{0} \Gamma_{0}\right], \text { etc. }
\end{aligned}
$$

where $d_{7}$ is given by $(36), H_{8} \cdots H_{10}$ are known functions of $D, h_{5} \cdots h_{8}$ are known non-zero constants and $F$ is another undetermined constant.

Equation (32) shows that

$$
\psi=\frac{1}{\gamma} f(\gamma R)+\frac{1}{\gamma^{2}} g(\gamma R) .
$$

(The first term is similar to that of Eq. (19) in §2.) In an exactly similar way to that used in $\$ 2$ we expand the $\psi$ of (32) by expressing $\gamma$ in terms of $\Gamma$ and expanding for large Re and $Z$ using (37), (39), (41), (42) and (43). Here one natural variable would appear to be proportional to $R \operatorname{Re}_{0} \Gamma_{0}$. However, keeping in mind the limit as the cylinder radius $a \rightarrow \infty$, but with $\left(r_{1}-a\right)$ finite, a more appropriate and equally valid variable is proportional to $R(R+2) \operatorname{Re}_{0} \Gamma_{0}$ 
or, in terms of $r,\left(r^{2}-1\right) \operatorname{Re}_{0} \Gamma_{0}$. If, for example, $C$ in (41) is zero we get comparable variables $\xi, \eta$ to those in (21) where here $\xi \propto{ }_{0} \Gamma_{0}^{-1}, \eta \propto\left(r^{2}-1\right) \operatorname{Re}_{0} \Gamma_{0}$, and $\xi, \eta$ are given as

$$
\xi=(\operatorname{Re} z)^{\frac{1}{2}}, \quad \eta=\frac{1}{4}\left(r^{2}-1\right)(\operatorname{Re} / z)^{\frac{1}{2}}
$$

or in dimensional form as

$$
\xi=\left(U z_{1} / \nu\right)^{\frac{2}{2}}, \quad \eta=\frac{r_{1}^{2}-a^{2}}{4 a}\left(\frac{U}{\nu z_{1}}\right)^{\frac{1}{3}} .
$$

The limit as $a \rightarrow \infty$ with $r_{1}-a \rightarrow y_{1}$ gives the limiting $\eta$ in $(44)$ as $\frac{1}{2} y_{1}\left(U / \nu z_{1}\right)^{\frac{1}{2}}$ which is the same as (21) with $z_{1}$ for $x_{1}$. With a similar transformation to (15) with $\zeta_{1}$ corresponding to $X_{1}$ we have

$$
\zeta_{1}=z_{1}+c U C_{1}(\nu)
$$

where $C_{1}(\nu)$ is the dimensional form of the undetermined constant $C$, we can write

$$
\xi=\left(U \zeta_{1} / \nu\right)^{\frac{1}{2}}, \quad \eta=\frac{r_{1}^{2}-a^{2}}{4 a}\left(\frac{U}{\nu \zeta_{1}}\right)^{\frac{1}{2}}
$$

in place of (44) (the special case of (45) with $C_{1}=0$ ). Equation (45) corresponds to (22).

The expansion of $\psi$ as described above using (24), (37), (39), (41), (42), (43), and (45) (or (44)) suggests the asymptotic form

$$
\begin{aligned}
\psi=\frac{\xi}{\operatorname{Re}}\left[f_{0}(\eta)\right. & \left.+\frac{1}{\xi^{2}}\left\{f_{2}(\eta)+g_{2}(\eta) \log \xi\right\}+\cdots\right] \\
& +\left(\frac{\xi}{\operatorname{Re}}\right)^{2}\left[F_{0}(\eta)+\frac{1}{\xi^{2}}\left\{F_{2}(\eta)+G_{2}(\eta) \log \xi\right\}+\cdots\right] \\
& +\left(\frac{\xi}{\operatorname{Re}}\right)^{3}\left[\mathscr{F}_{0}(\eta)+\frac{1}{\xi^{2}}\left\{F_{2}(\eta)+\mathcal{S}_{2}(\eta) \log \xi\right\}+\cdots\right]+\cdots
\end{aligned}
$$

The higher order terms in each square bracket suggested by the method here are $O\left((\log \xi)^{2} / \xi^{4}\right)$ as in $\$ 2$. However, with the experience from that case the higher order terms are probably $O\left((\log \xi)^{2} / \xi^{3}\right)$ and so the extension to each of the coordinate expansions in (46) is probably of the same form as the correct one for the semi-infinite flat plate case discussed in $\$ 2$. Thus the probable extension to the first bracket in (46) is

$$
\frac{1}{\xi^{3}}\left\{f_{3}(\eta)+g_{3}(\eta) \log \xi+h_{3}(\eta)(\log \xi)^{2}\right\},
$$

with similar ones for the higher $(\xi / \mathrm{Re})$-terms.

$\dagger$ There is still the possibility that arbitrary multiples of eigensolutions of $L_{n} f_{n}=0$ (see (50)) for non-integral $n$ might be required in this equation within each square bracket (see the footnote after (23) as well). These solutions for such $n$ have double zeros at $\eta=0$ and tend to zero exponentially for $\eta \rightarrow \infty$. 
The solution suggested by (46) is self-similar in the sense that ordinary differential equations are required to be solved for the functions of $\eta$ as shown in $\$ 4$. The equations (25) and (26) do not possess similarity solutions in the same sense that the boundary layer equations do. The similarity variables used with the boundary layer equations are $\xi / \operatorname{Re}$ and $\eta$. These were first introduced by Atkinson and Goldstein (see Goldstein (1938)) and used by subsequent workers. The solution (46) has in effect three 'variables' $: \xi, \xi / \mathrm{Re}, \eta$.

4. The ordinary differential equations for $f_{0}, f_{2}, g_{2}, F_{0}, \cdots$ and the skin friction on the cylinder. The functions of $\eta$ in (46) are determined from the ordinary differential equations obtained by substituting (46) into the full Navier-Stokes equations (25) and (26) in vorticity form and equating the coefficients of powers of $\xi$ in the usual way. The following ordinary differential equations are obtained:

$$
\begin{aligned}
f_{0}^{\prime \prime \prime}+f_{0} f_{0}^{\prime \prime} & =0, \quad L_{2}\left(g_{2}\right)=0 \\
L_{2}\left(f_{2}\right) & =d\left(\eta f_{0}^{\prime}-f_{0}\right)^{2} / d \eta+\left(f_{0}^{\prime} g_{2}^{\prime \prime}-f_{0}^{\prime \prime \prime} g_{2}\right) \\
L_{-1}\left(F_{0}\right) & =-4\left(2 f_{0}^{\prime \prime \prime}+\eta f_{0}^{\mathrm{iv}}\right) \\
L_{1}\left(G_{2}\right) & =-\left(2 F_{0} g_{2}^{\prime \prime \prime}+3 F_{0}^{\prime} g_{2}^{\prime \prime}+F_{0}^{\prime \prime} g_{2}^{\prime}+8 g_{2}^{\prime \prime \prime}+4 \eta g_{2}^{\mathrm{iv}}\right) \\
L_{1}\left(F_{2}\right) & =f n\left(f_{0}, g_{2}, f_{2}, F_{0}, G_{2}\right) \\
L_{-2}\left(\Im_{0}\right) & =-2\left(F_{0} F_{0}^{\prime \prime \prime}+4 F_{0}^{\prime \prime \prime}+2 \eta F_{0}^{\mathrm{iv}}\right) \\
L_{0}\left(g_{2}\right) & =-\frac{d}{d \eta}\left(3 F_{0} g_{2}^{\prime \prime}-\Im_{0}^{\prime \prime} g_{2}+2 F_{0} G_{2}^{\prime \prime}+4 G_{2}^{\prime \prime}+4 \eta G_{2}^{\prime \prime \prime}\right) \\
L_{0}\left(F_{2}\right) & =f n\left(f_{0}, g_{2}, f_{2}, F_{0}, G_{2}, F_{2}, F_{0}, G_{2}\right)
\end{aligned}
$$

where the operator

$$
L_{n}=\frac{d^{4}}{d \eta^{4}}+f_{0} \frac{d^{3}}{d \eta^{3}}+(n+1) f_{0}^{\prime} \frac{d^{2}}{d \eta^{2}}+f_{0}^{\prime \prime} \frac{d}{d \eta}-(n-1) f_{0}^{\prime \prime \prime} ;
$$

and where the notation $f n$ is used to show the functional dependence.

There appears, from (46)-(50), to be a pattern within each coordinate series in successive $\xi / \operatorname{Re}$ terms in (46) in that the first (zeroth) functions satisfy an equation with $n=n_{1}$, say, in $L_{n}$, the second and third terms satisfy equations with $n=n_{1}+2$ in $L_{n}$, the fourth, fifth and sixth with $n=n_{1}+3$, and so on. Further, higher $\xi / \operatorname{Re}$ terms seem to have a value of $n$ one less than the term previous to it (see (47), (48), (49)). It seems, at this stage, that the operator $L_{n}$ will appear in this solution with all positive and negative integral values of $n$. The semi-infinite flat plate solution ( $\$ 2$ ) has only positive integral values of $n$ in $L_{n}$, but the possibility of non-integral $n$ does exist.

The boundary conditions for (47)-(49) are that each function and its first derivative must be zero at $\eta=0$. The remaining conditions must be given for large $\eta$ by the form of the external flow into which $\psi$ from (46) must merge, for large $\eta$, with exponentially small vorticity. The conditions on $f_{0}, f_{2}, g_{2}, \cdots$ and 
the computed functions have been given by Goldstein (1960) and Murray (1965a). Until the potential flow into which higher $\xi / \mathrm{Re}$ terms must merge, is found, the outer (large $\eta$ ) boundary conditions cannot be specified with any confidence. Obtaining the external potential flow and hence the $F_{0}, F_{2}, G_{2}, \cdots$, $F_{0}, F_{2}, \mathcal{G}_{2} \ldots$ is not the purpose of this paper. However, even without it, certain observations can be made about the solution given by (46).

The operator $L_{n}$ can be integrated once to give $K_{n}$ where

$$
K_{n}=\frac{d^{3}}{d \eta^{3}}+f_{0} \frac{d^{2}}{d \eta^{2}}+n f_{0}^{\prime} \frac{d}{d \eta}-(n-1) f_{0}^{\prime \prime} .
$$

If the constant of integration is set equal to zero then the equations resulting for $F_{0}$ and $F_{0}$ (and $f_{0}$ ) are the same as those given by Goldstein (1938) for the entry flow into a circular pipe if $\eta$ and the functions have reversed signs to those above and if the constants in that problem which relate to the constant pipe flow condition are set equal to zero. This first integral form with $K_{n}$ was given by Goldstein (1960) and it is useful in obtaining asymptotic forms for the solutions of $(47)-(49)$.

The dimensionless skin friction coefficient $\tau$ on the cylinder is given by

$$
\tau=(a / \mu U)\left[\mu w_{1 r_{1}}\right]_{r_{1}=a}=\frac{1}{4}\left(\frac{\operatorname{Re}}{\xi}\right)^{2}\left[\psi_{\eta \eta}\right]_{\eta=0},
$$

where $\mu$ is the viscosity. From (46) we get

$$
\begin{aligned}
\tau=\frac{1}{4}\left(\frac{\operatorname{Re}}{\xi}\right)\left[\left[f_{0}^{\prime \prime}(O)+\left(\frac{\xi}{\operatorname{Re}}\right) F_{0}^{\prime \prime}(O)+\frac{\log \xi}{\xi^{2}} g_{2}^{\prime \prime}(O)\right.\right. \\
\left.+\left(\frac{\xi}{\operatorname{Re}}\right)^{2} F_{0}^{\prime \prime}(O)+O\left(\frac{1}{\xi^{2}}\right)\right] .
\end{aligned}
$$

In (52), $f_{0}$ is the Blasius solution, $F_{0}, F_{0}$ have been computed by Seban and Bond (1951) and Kelly (1954) and $g_{2}$ has been given by Murray (1965a). In dimensional form the skin friction coefficient $\tau_{1}$ is then given by

$$
\begin{aligned}
\frac{a \tau_{1}}{\mu U}=0.332\left(\frac{U a^{2}}{\nu z_{1}}\right)^{\frac{1}{3}}+0.696+0.551\left(\frac{a}{z_{1}}\right) & \left(\frac{\nu}{U z_{1}}\right)^{\frac{1}{2}} \log \left(\frac{\nu}{U z_{1}}\right)^{\frac{1}{2}} \\
& -0.797\left(\frac{\nu z_{1}}{U a^{2}}\right)^{\frac{1}{2}}+O\left(\frac{\nu a^{2}}{U z_{1}^{3}}\right)^{\frac{1}{2}},
\end{aligned}
$$

where $\xi$ as given by (44) has been used so that comparison can be made with previous solutions. If (45) were used in place of (44) the $z_{1}$ would simply be replaced by $\zeta_{1}$. An alternative way of writing (53) which shows more clearly the role of the Reynolds number based on $z_{1}$ is

$$
\begin{aligned}
\frac{a \tau_{1}}{\mu U}=0.332\left(\frac{U z_{1}}{\nu}\right)^{\frac{1}{2}}\left(\frac{a}{z_{1}}\right)+ & 0.696+0.551\left(\frac{\nu}{U z_{1}}\right)^{\frac{1}{2}}\left(\frac{a}{z_{1}}\right) \log \left(\frac{\nu}{U z_{1}}\right)^{\frac{1}{2}} \\
& -0.797\left(\frac{\nu}{U z_{1}}\right)^{\frac{1}{2}}\left(\frac{z_{1}}{a}\right)+O\left(\left(\frac{\nu}{U z_{1}}\right)^{\frac{1}{2}}\left(\frac{a}{z_{1}}\right)\right) .
\end{aligned}
$$


To date the solution which has been used for comparison with solutions obtained by approximate methods consists of the first, second and fourth terms in (53). It is clear from (53) or (54), that the third term involving $\left(\nu / U z_{1}\right)^{\frac{1}{2}}$ $\log \left(\nu / U z_{1}\right)^{\frac{1}{3}}$ is larger than the fourth in the region of interest. Furthermore, in the range where $z_{1} / a$ is comparable to $a / z_{1}$ the fourth term will be affected by the $O\left(\left(\nu / U z_{1}\right)^{\frac{1}{2}} a / z_{1}\right)$ term in $(54)$. This term however, comes from $f_{2}^{\prime \prime}(0)$ which as shown by Goldstein (1960) and Murray (1965a) (and suggested by this method in $\$ 2$ above) contains an undetermined constant. Thus, the fourth term cannot be given exactly at this stage.

Thus, to summarize, the solution for the skin friction coefficient where the boundary layer is thin compared with the cylinder radius is given by (53) or (54). To continue this solution into the region where the boundary layer is large compared with the radius Glauert and Lighthill's (1955) solution should be used. Their solution is not valid over the complete length of the cylinder as suggested since the $\nu \log v$ is absent from their solution in the range discussed in this paper. The suggested curves for the skin friction, drag and displacement area as given by Glauert and Lighthill (1955) (and reproduced by Rosenhead (1963) and others) are valid in the low $\left(\nu z_{1} / U a^{2}\right)$-, or $\left(\nu / U z_{1}\right)\left(z_{1} / a\right)^{2}$-range only up to the second term. For higher order accuracy a logarithmic term as in (53) or (54) must be incorporated. The same remarks will apply to Varley (1957), Cooke (1957) and others for non-circular cylinders. In the limit as $a \rightarrow \infty,(52)$ (and (53)) give the skin friction for the semi-infinite flat plate as found by Goldstein (1960) and Murray (1965a) while the other solutions discussed above do not.

To return to (47)-(49), the operator $L_{n}$ in (50) and $K_{n}$ in (51) appear repeatedly in problems of a semi-infinite nature and one infers that perhaps it is fundamental in solutions of the Navier-Stokes equations. For example $L_{n}, K_{n}$, $n \geqq 2$, occur in the Goldstein (1960) fundamental flat plate solution. $L_{-1}$ occurs in the case of a semi-infinite flat plate in a uniform shear (see Murray (1961)) and also in slip flow past a semi-infinite flat plate (see Murray (1965b)). In the cylinder problem here $L_{n}, K_{n}$ appear for all positive and negative integral $\uparrow n$. A connection between $n$ and the power of $\xi, m$, say, occurring in (46) is that $m+n=1$. The first term is, of course, omitted from this, it being the coefficient in the $L_{n}$. If $n$ is not restricted to integral values the operator $K_{n}$ in (51) appears in perturbations about the Blasius equation (the first of (47)). Perturbations about the Blasius equation have been studied by Libby and Fox (1963): that is, they studied solutions of an equation $K_{n} f=0$ where $n$ is now an eigenvalue. In a sense, then, terms which improve on the Blasius solution in the flat plate solution and others including the above may be thought of in terms of integral perturbations about the Blasius solution. This does not cover the above solution, however, since such perturbations as studied by Libby and Fox are restricted to complimentary functions of the integrated form of $L_{n}$ only. The inclusion of non-integral terms in the $\psi$ 's of (23) and (46) is of course a real possibility.

† Certain non-integral $n$ may also appear. 
5. Conclusions. The method suggested here for obtaining asymptotic forms for a class of Navier-Stokes solutions is to start with an approximate solution of the Oseen boundary layer equations, modify this solution by replacing $U$ by a function of $U, \nu$ and the axial coordinate, and require the resulting form to satisfy an integral of the Navier-Stokes equation with this modified solution inserted. This results in an ordinary differential equation for the function replacing $U$ and the solution is found in asymptotic form in general. The method seems to be fairly successful. Its application to flow past a semi-infinite flat plate gave the form of the first three terms correctly and indicated the type of singularity that could be expected in higher terms. The possible origin displacement is also predicted by the method: the actual effective displacement is unknown at this stage since the leading edge solution has not yet been found.

The application to axial flow past a circular cylinder resulted in a solution which differs from those previously obtained using the boundary layer equations. This is not, of course, surprising, but it also showed that a term $O\left(\nu^{\frac{1}{2}} \log v\right)$ is the probable third term in the asymptotic solution for the skin friction coeffcient, etc. and not simply $O\left(\nu^{\frac{1}{2}}\right)$ as previously suggested. The correct flat plate limit is given as the limiting case of the cylinder radius tending to infinity. In view of this solution for the circular cylinder the suggested curves for the skin friction coefficient, drag, etc. as given by Glauert and Lighthill (1955), Rosenhead (1963), Varley (1957), Cooke (1957) and any results based on them are probably not valid beyond the $O(1)$ terms in their series form used to compute them in the region near the leading edge. The results given here and those given recently by Murray (1965b) in the case of slip flow past a semi-infinite flat plate suggest that it is usually unprofitable to study higher order solutions of the boundary-layer or the Oseen equations $t$ in an attempt to get closer approximations to the Navier-Stokes solution since fundamentally different singularities exist in the latter as compared with the former. The solution, or form of it, given here is only a suggestion at this stage. To complete the problem an external potential flow must be found and the functions of $\eta$ in $\psi$ given by (46) must be shown to meet the necessary requirements to allow the solution near the cylinder to merge into the potential flow with exponentially small vorticity.

It is hoped to pursue the study of this method initially to fluid mechanical problems of a more general nature by using an Oseen boundary layer solution as a starting point. In the semi-infinite flat plate problem an attempt was made to use the Blasius boundary layer solution with a modified $\eta$ in place of the error function but without success. The importance of being able to use a first order singular perturbation solution in place of an Oseen-type solution as a type of generating function in singular perturbation problems in general is clear. A study along these lines is also envisaged.

\section{REFERENCES}

BATCHeLor, G. K. The skin friction on infinite cylinders moving parallel to their length. Quart. J. Mech. App. Math. 7, 179, 1954.

† This is particularly so with semi-infinite bodies, of course. 
CARRTER, G. F. Analytic approximation techniques in Applied Mathematics. J. Soc. Indust. App. Math. 13, 68, 1965.

Cooke, J. C. The flow of fluid along cylinders. Quart. J. Mech. App. Math. 10, 312, 1957.

Glauert, M. B. AND Lighthill, M. J. The axisymmetric boundary layer on a long thin cylinder. Proc. Roy. Soc. A, 230, 188, 1955.

Goldstein, S. Modern Developments in Fluid Dynamics. Oxford: Clarendon Press, 1938.

Goldstein, S. Flow of an incompressible viscous fluid along a semi-infinite flat plate. Inst. Engng. Res., Univ. Calif., Tech. Rep. HE-150-144, 1956.

Goldstein, S. Lectures on Fluid Mechanics. New York: Interscience, 1960.

KeLLX, H. R. A note on the laminar boundary layer on a circular cylinder in axial compressible flow. J. Aero. Sci. 21, 634, 1954.

Lewis, J. A. And Carrier, G. F. Some remarks on the flat plate boundary layer. Q. App. Math. 7, 228, 1949.

LIBBy, P. A, AND Fox, H. Some perturbation solutions in laminar boundary layer theory. Part 1. The momentum equation. J. Fluid Mech. 17, 433, 1963.

MURrax, J. D. The boundary layer on a fiat plate in a stream with uniform shear. J. Fluid Mech. 11, 309, 1961.

Murrat, J. D. Incompressible viscous flow past a semi-infinite flat plate. J. Fluid Mech. $21,337,1965 \mathrm{a}$.

Murray, J. D. Incompressible slip flow past a semi-infinite flat plate. J. Fluid Mech. 22, $463,1965 \mathrm{~b}$.

Rayleigh, LoRd. On the motion of solid bodies through viscous liquids. Phil. Mag. (6), 21, 697, 1911.

Rosenhead, L. Laminar Boundary Layers, Oxford: Clarendon Press, 1963.

Seban, R. A. ANd Bond, R. Skin friction and heat transfer characteristics of a laminar boundary layer on a cylinder in axial compressible flow. J. Aero. Sci., 18, 671, 1951.

Sowerby, L. And Cooke, J. C. The flow along corners and edges. Quart. J. Mech. App. Math., 6, 50, 1953.

Stewartson, K. The asymptotic boundary layer on a circular cylinder in axial incompressible flow. Quart. App. Math., 13, 113, 1955.

Van Drke, M. Perturbation Methods in Fluid Mechanics. New York: Academic Press, $1964 a$.

VAn Drke, M. Higher approximations in boundary-layer theory. Part 1. General Analysis. J. Fluid Mech., 14, 161, 1962a.

VAN Dyre, M. Higher approximations in boundary-layer theory. Part 2. Application to leading edges. J. Fluid Mech., 14, 481, 1962b.

VAN DYKE, M. Higher approximations in boundary-layer theory. Part 3. Parabola in uniform stream. J. Fluid Mech., 19, 145, 1964b.

VARLEY, E. An approximate boundary-layer theory for semi-infinite cylinders of arbitrary cross-section. J. Fluid Mech., 3, 601, 1958.

Weinbaum, S. Natural convection in a horizontal circular cylinder. J. Fluid Mech., 18, 409, 1964.

The University of Michigan

ANn ARbor, Michigan

(Received October 18, 1965) 